\title{
LA VIVENCIA DE UN GRUPO DE JUBILADOS-ANCIANO: INVESTIGACIÓN - ACCIÓN
}

Rosalina Aparecida Partezani Rodrigues* Maria Manuela Rino Mendes*

Maria Lúcia Zanetti* Maria Suely Nogueira* Lina Maria de Oliveira Azoubel** Ana Cecília Chaguri***

RODRIGUES, R.A.P.; MENDES, M.M.R.; ZANETTI, M.L.; NOGUEIRA, M.S.; AZOUBEL, L.M.O.; CHAGURI, A.C. La vivência de un grupo jubilado-anciano: investigación - acción. Rev.latino-am.enfermagem, Ribeirão Preto, v. 5, n. 4, p. 85-89, outubro 1997.

Este estudio se propone comprender la situación del jubilado, a través de la vivencia en grupo, y permitir que los jubilados refleccionen con respecto a su condición. Se ha utilizado la metodologia de la investigación - acción, con 25 sujetos jubilados de la Universidad, con edad entre 51 y 79 años. Las narraciones respecto de las vivencias fueron obtenidas por medio de interacción verbal, discusión en grupo y entrevista. En el análisis de las vivencias han emergido cuestiones relacionadas a los intereses de los jubilados ancianos, cuanto a la salud física y mental, relacionamiento, aprendizaje, ambiente social y ocio.

TÉRMINOS CLAVES: anciano, jubilados, grupos de vivencia

\section{INTRODUCCIÓN}

La transición demográfica que se evidencia en los paises desarrollados, a partir de la década del 50 en el grupo de la población con 60 años o más de edad; se expresa también en los paises en desarrollo, en las últimas décadas de este siglo. Existen estimativas en cuanto al rápido crecimiento de los ancianos teniendo como comparación los demas intervalos de edad.

La población anciana en Brasil está estimada en 10 millones de habitantes, que lo sitúa entre los 10 primeros paises del mundo. Aunque el envejecimiento problacional sea analizado bajo las variantes: mortalidad y fecundidad, debe ser considerada, la migración interna, que en Brasil explica un mayor número de ancianos en las regiones urbanas y en los polos sudeste y sur.

Los últimos censos de demografía brasileños vienen presentando tendencia al crecimiento de la expectativa de vida de la población y por consiguiente un mayor número de personas irá ha constituirse en la condición de jubilado.

La Constitución actual delibera algunos beneficios y servicios de asistencia médica para los jubilados, entre ellos la jubilación y las pensiones.

Los jubilados enfrentan sérios problemas con la desvinculación del proceso productivo, reflejo de una política social sin compromiso con la población que ha trabajado árduamente en la construcción de este país. Se evidencia salarios bajos, servicios de asistencia médica deficiente, aspectos estos que desencadenan la marginalidad de los jubilados y ancianos.

La condición de la población que usufructu de buenos salarios y posee otras fuentes de renta, resultado de la acumulación de bienes obtenidos en su vida de trabajador, no es objeto de estudio, porque ésta configúrase como una excepción de la pobreza generalizada en que viven jubilados y ancianos.

La jubilación se ha tornado difícil para la población de baja renta, una vez que el beneficio recibido de la Asistencia Social no consigue suplir las mínimas necesidades de vida, la sociedad no ofrece opciones alternativas de alistamiento y aprovechamiento de su potencial.

Implicaciones en la vida de los jubilados con la

\footnotetext{
* Profesor Doctor del Departamento de Enfermería General y Especializada de la Escuela de Enfermería de Ribeirão Preto de la Universidad de São Paulo - Brasil

** Profesor Asistente del Departamento de Enfermería Maternoinfantil e Salud Publica de la Escuela de Enfermeria de Ribeirão Preto de la Universidad de São Paulo - Brasil

*** Psicóloga del Nucleo de Investigación en Gerontología de la Escuela de Enfermería de Ribeirão Preto de la Universidad de São Paulo - Brasil
} 
pérdida del rol "ser trabajador" son expresadas en la sociedad a través de un estereotipo asociable entre la jubilación, vejez y miseria. Este cuadro contribuye para la ruptura en la vida de los jubilados perdiendo su prestigio entre amigos y familiares, diseminando la sensación de inutilidad con el rebajamiento del nivel económico.

Fundamentadas en la comprensión de que la jubilación en Brasil desencadena la exclusión social caracterizada por el desprestigio, desinterés, depresión, soledad, pobreza y por estar inmotivado, esto nos lleva a considerar que dicha situación suscita cuestionamientos en cuanto a los roles y valores de las personas en la sociedad.

Una de las maneras de buscar comprender la situación de la jubilación y vejez, en una determinada sociedad, puede ser la realización de la investigación acción que permita vivenciar en grupo de personas en una referida condición.

La formación de grupos, según ROGERS (1979) "es la invención social", que se ha difundido más rápidamente en este siglo. El avance en el movimiento de la organización de grupos, ha se evidenciado en las más distintas instituciones y situaciones. Destacamos entre ellas la Universidad, preocupada en observar, describir, comprender y discutir las transformaciones que se verifican en la sociedad, así como contribuir en la producción de conocimiento.

"Grupo de encuentro" es una modalidad de movimiento en que se "acentúa el crecimiento personal y el desenvolvimiento y perfeccionamiento de la comunicación y relaciones interpersonales, a través de un proceso experimental" (ROGERS, 1979).

La Escuela de Enfermería de Ribeirão Preto de la Universidad de São Paulo, en sus Departamentos adopta la estrategia de formación de grupos para realizar con viabilidad sus finalidades fundamentales de enseñanza, investigación y extensión de servicios a la comunidad, y tambien en cuestiones de burocrácia.

En el presente estudio nos proponemos describir a través de la investigación-acción la situación de los jubilados-ancianos, en grupo y permitir que ellos reflexionen con respecto a su condición de vida.

\section{MÉTODO}

La investigación-acción con el grupo de jubilados y ancianos posibilita la descripción de la situación relatada por sus miembros, la identificación de sus dificultades, así como las iniciativas para proceder e intentar solucionarlas, construyéndose el conocimiento con respecto a la condición del jubilado.

La investigación-acción, según THIOLLENT (1988), consiste en la posibilidad de tornar viable dos tipos de intenciones: el práctico, que permite, lo mejor posible del ecuacional del problema, considerando central la investigación y lleva a acciones y propuestas de soluciones; y el de cuño teórico, que visa obtener conocimiento adoptando procedimientos conforme las diversas situaciones vivenciadas.

El processo de esa investigación no exige un seguimiento riguroso de fases, no obstante recurrre un camino que parte de la exploración a la definición de objetivos y planes para la acción, llegando a la divulgación de los resultados, en un movimiento constante de acción-reflexión.

La participación de los integrantes del grupo en una investigación-acción debe ser incentivada por el investigador, sabiendo anticipadamente que podrá encontrar situaciones de mayor o menor participación y envolvimiento. Destacandose la indecisión, resistencia a la expresión, la expresión de sentimientos pasados, expresión de sentimientos interpersonales, aceptación y principio de los cambios, que interfieren en las tentativas para solucionar los problemas encontrados (ROGERS, 1979).

La investigación-acción fué realizada junto a un grupo de jubilados de la Universidad de São Paulo, por un grupo de profesionales de enseñanza: enfermeros, nutricionistas, psicólogos y de educación física.

El primer momento del trabajo fue hacer una estadística numérica y nominal de dirección de jubilados de los últimos cinco años, en el Campus de la Universidad de São Paulo. El segundo paso fué la invitación hecha por escrito, encaminada por correo, para jubilados ancianos, para participar de un grupo de encuentros, haciendo constar el objetivo de este y solicitarles la respuesta cuanto al interés.

Planeamos para el primer encuentro:

- identificar los aspectos de interés de los jubilados utilizando la técnica de interacción en grupo;

- anotar las contribuciones de los miembros en carteles, diários de campo, fotografías y video;

- establecer día y periodo para los encuentros del grupo en la Escuela de Enfermería de Ribeirão Preto Universidad de São Paulo.

Los encuentros posteriores fueron utilizados para direccionar el curso de las propuestas de convivencia en el grupo, narraciones de otras vivencias y memorias de los jubilados y ancianos, después de un año de la formación del grupo.

Para la comprensión de la situación del jubilado anciano serán descritas las estrategias de operacionalización, al fin de un período de encuentros. A través de entrevistas formales, realizadas por los investigadores con los sujetos, se puede también obtener otras importantes narraciones.

Los temas fueron identificados, organizados en un sistema de categorías de conformidad con el significado de las palabras. 


\section{ANALISIS Y DISCUSION DE LOS DATOS}

\section{Caracterización de los sujetos}

La relación de jubilados del Campus de Ribeirão Preto - Universidad de São Paulo fue obtenida junto a las otras Unidades, con un total de 400 personas, las cuales solamente el $10 \%$ contestó la invitación.

Este grupo se constituyó con 25 sujetos, en edades entre 51 y 79 años, siendo que el $76 \%$ son del sexo femenino. De estos el $60 \%$ son católicos, $20 \%$ espíritas, $16 \%$ sin religión definida y el $4 \%$ son protestantes.

En cuanto a la cuestión de nivel escolar, el predominio es de $36 \%$ con el primaria completa.

En relación al estado civil, $52 \%$ son casados, $24 \%$ viudos, $8 \%$ solteros, $4 \%$ viudos con compañeros, $4 \%$ anulados y $4 \%$ separados. En su totalidad viven con personas de la família, y el $76 \%$ viven en casa propia.

Del total de los sujetos investigados, $88 \%$ son jubilados; la mayoría por tiempo de servicio, seguido de invalidez.

La remuneración de la jubilación está entre 90 y 180 dólares norte americanos, con una renta familiar mensual entre 90 y 1200 dólares.

Vale resaltar que los jubilados que reciben mayor renta en la Universidad y de mayor nivel de instrucción no contestaron la invitación. Este hecho puede ser debido a la participación de los ancianos en otras inserciones sociales. Otro aspecto fundamental puede ser el estereotipo en cuanto al agrupamiento de un cierto número de personas jubiladas.

\section{Estrategia de operacionalización de las vivencias}

El grupo de vivencias se ha desarrollado bajo un proceso de interacción grupal utilizando las distintas maneras de comunicación, que al ser analizadas, se ha notado el secuencial de las fases citadas por ROGERS(1979).

\section{$1^{\text {o) }) ~ I n d e c i s i o ́ n ~}$}

El Coordinador del Grupo, esclareció que el primer encuentro se trataba de una reunión informal. En este encuentro se ha estimulado el relacionamiento entre los jubilados ancianos aplicándose la técnica caracol, seguida de discusión en grupo para incentivar la expresión de sus intereses. Se ha notado que los participantes sondeaban el ambiente, permanecían mirando con atención, pocas palabras, algunas lagunas.

\section{$\left.2^{\circ}\right)$ Resistência a la expresión}

En esta fase los jubilados ancianos, estimulados por el uso de técnicas de discusión en grupo empezaron a expresar pensamientos, valores provenientes del interior de cada uno y de las interacciones con las personas involucradas. La resistencia ha variado de persona a persona, unos son descontraídos, abiertos; encuanto otros son muy cerrados en si mismos. La revelación de la expresión de cada uno fue emanada en la medida que el grupo iba ampliando las oportunidades de convivencia donde revelaban sus sentimientos frente a comportamientos en distintos momentos.

\section{$\left.3^{\circ}\right)$ Expresión de sentimientos pasados}

Ha emergido en los momentos en que la discusión se hacía en torno del trabajo y de las relaciones familiares. Trátase de un grupo de personas que tuvieron relacionamientos en el trabajo y muchos recuerdos de vivencia fueron reveladas en los diversos encuentros.

Una anciana comenta: "yo me siento frustrada por no haber sido aprobada en el exámen de admisión para la Faculdad de Medicina". Otra todavía, revela que: "tuve sérios problemas con la jefatura de mi trabajo". Otro ejemplo es que durante una actividad corporal con música, en un ambiente natural, una de las ancianas se puso a llorar y dijo: "cuando me encuentro en contato con la naturaleza, me recuerdo de la fase terminal de mi esposo, cuando yo no tenía con quien hablar y angustiada yo salía para el parque y conversaba con la naturaleza".

\section{$\left.4^{\circ}\right)$ Expresión de sentimientos interpersonales}

La relación interpersonal permite que las personas se expresen de distintas maneras, entre ellas la verbal y la no verbal.

Una anciana reveló: "las personas que deben participar de un grupo, tienen que ser seleccionadas", haciendo referencia a una anciana que no es muy bien aceptada en el grupo, por su manera de ser. Otras, todavía, dicen que la convivencia en grupo, ha posibilitado un mayor número de amigos, de ambos sexos y de una mejor conducta con sus familiares y vecinos.

\section{$\left.5^{\circ}\right)$ El desarrollo de una capacidad terapéutica en el grupo}

Los jubilados - ancianos tienen una característica que marca; la mayoría participa del grupo para ser ayudado y otras veces para ayudar. Han ocurrido varias situaciones que ejemplifican los efectos del grupo. La Sra. María viene presentando disturbios de comportamiento, como: soledad, depresión; y los miembros del grupo han trabajado en una dinámica que apoya el encuentro de las personas en el própio grupo y afuera del él; la Sra. María se ha beneficiado de esa 
interacción. Ha estimulado también la formación de lo que puede ser articulado en grupo, en la sociedad.

\section{$\left.6^{\circ}\right)$ Aceptación y comienzo de cambio}

Puede ser evidenciado en esta fase niveles diversificados, en algunos ancianos en las distintas actividades de grupo. Esta constatación ha motivado a los investigadores a elaborar un instrumento de recolección de datos que permitiese la expresión de los ancianos frente a las vivencias del grupo.

\section{$7^{\circ}$ ) Vivencia del grupo de jubilados ancianos}

Durante el proceso de desarrollo del grupo, sentimos la necesidad de caracterizarlo, identificando los intereses, expectativas, describiendo la participación, contribución y apreciando a los jubilados, a través de un instrumento formal.

En lo que se refiere a los intereses, fué posible caracterizarlos en cuanto a la salud mental y física, al relacionamiento, al aprendizage y a la prevención de la vejez.

Los intereses y expectativas expresadas reflejen la percepción de los problemas de soledad, depresión, ausencia de diálogo, reclusión y la búsqueda de ambiente social para reencuentro, comunicación, convivencia, ocio $\mathrm{y}$ desarrollo de las actividades que valoren su vida. Estos aspectos pueden ser evidenciados en las declaraciones "para no quedar clausurados en casa y no envejecer mas rápido", o en otras como: "es estar con otras personas, expandirse más, es importante para la salud, cuidarse mejor, aprender más en la vida". Otro todavía cuenta que es bueno "tener ideas nuevas, nuevas amistades y conocimiento". En cuanto a la participación de los jubilados, relatan que necesitan de nuevos aprendizajen, relacionamientos e incentivo al ambiente social.

Conforme expresan algunos participantes, el grupo de ancianos "trajo una motivación, desarrollando cambios en la dinámica de mi vida", así como, "nos ayuda, disipa preocupaciones, las personas colocan sus ansiedades y solan fuera sus sufrimientos".

Otra cuestión es como el jubilado se siente al participar del grupo. Las narraciones han mostrado mayor relacionamiento, salud mental, mejoría de la salud física, ampliación del ambiente social y motivación.

Para este grupo, el relacionamiento es más alegre "porque uno se suelta más, conversa más" y porque "ha cambiado mi vida, y que los compañeros que he encontrado me traen muchas recordaciones".

La cuestión de la salud mental está muy presente en este grupo, porque "lo que sentía: como dolor en el estómago, dolor de cabeza, parece que todo ha desaparecido", y que "no necesito tomar mas remédios, es una terapeútica excelente para mí, todavía reconozco que "antes el trabajo me parecía molestar, hoy consigo hacer los deberes, más en orden".

Verdaderamente "hago más ejercícios y me preocupo conmigo", hasta "estoy entusiasmada para hacer dieta", pues el grupo "ayuda a valorar nuestra vida".

Otro aspecto es la contribución del grupo para el jubilado cuanto a la independencia, enfrentando la vida y los relacionamientos. Sabemos de antemano que fue corto el período de convivencia ( 9 meses) para poder evaluar cambios de orden, considerando que los cambios en los adultos y ancianos son mas detenidos, enfrentan más restricciones.

En la independencia "sentí más libertad", "está mejor porque sali de casa", "en fin, estoy más libre, me relaciono más", “cuando camino por las calles, las personas me miran de otra manera", y también vale recordar que "me siento más joven, me arreglo, me gusta charlar, cambiar experiencias".

Uno de ellos reconoce que la contribución del grupo en cuanto el enfrentar es "encarar la realidad". Algunos jubilados, todavía, dícen que no ocurrieron alteraciones. Lo que se nota, es que muchas veces las personas no concientizan sus alteraciones internas. Es necesario pensar más en estas cuestiones, aumentar la búsqueda del acto de uno reflejarse para conseguir verbalizar las alteraciones que ocurrieron. Otro todavía cita que "ha cambiado para mi, porque mi señora, que también participa del grupo es muy exigente y como miembro del grupo ha cambiado como persona y nuestro relacionamiento ha mejorado".

Nótese en las narraciones, que el grupo ha posibilitado a los ancianos la participación en las distintas actividades direccionadas para la salud física, ocio y relacionamiento. Todavía, al trabajar con história de vida, a través de fotografías y de la comunicación verbal, verificamos que una de las ancianas ha revelado "la única cosa que no me gustó fue la de la história de la vida (con fotos)". Esta técnica hizo emerger sentimientos tristes del pasado, en el grupo, de recuerdos vividos y que todavía estaban presentes.

Las intenciones en participar del grupo fueron expresadas asi: "hace parte de mi vida", "yo me siento bien", "el grupo me da amor", todavía sugieren que "debe haber más juegos, conmemoraciones de cumpleaños, trabajos de artesanía", y oportunidad para mostrar sus capacidades artísticas.

Los jubilados ancianos sugieren además que la actividad física, la ludoterapéutica, las conferencias y los paseos son temas considerados importantes en la continuidad del programa. Dicen dar énfasis sobre la gimnasia con más frecuencia "me gustaría tener más 
clases de gimnasia", así como la hidrogimnasia y las caminatas.

La ludoterapéutica expresada como "trabajos manuales, costura, juegos, pintura en tejidos", es una actividad que permite una mayor relación entre el grupo. Los paseos de nivel cultural también fueron evidenciados y las conferencias direccionadas para el envejecimiento más saludable.

El alistamiento en la jubilación lleva el jubilado a perder sus valores personales de estima, imagen, demostrando a través de relacionamientos en el grupo algunas pérdidas de convivencia en la sociedad.

\section{CONCLUSIONES FINALES}

La investigación - acción ha permitido la configuración de un grupo de jubilados ancianos que cultivaron un espacio de exponerse, de conocerse, de convivir y ampliar su círculo de amistades interfiriendo en su condición de vida, rescatando aspectos que se perdieron con el proceso de envejecer, y de desencajamiento del trabajo.

A pesar de ser nuestra primera experiencia en grupo de vivencia, percibimos que este es un camino que permite la posibilidad para el entendimiento del fenómeno de la jubilación y vejez, y la búsqueda de entendimiento y principio filosófico y teórico para el análisis del fenómeno e intervención en ese proceso.

\section{RECOMENDACIONES}

Con fundamento en esa experiencia, proponemos:

1) la formación de nuevos grupos de vivencia con ancianos-jubilados,

2) que pueda ocurrir cambio de experiencias entre los referidos grupos, con el objetivo de enriquecer el trabajo con los ancianos y los coordinadores, y

3) que se implementen nuevas maneras de grupos de vivencia con diferentes estrategias.

4) que se investigue la adaptación a la jubilación y vejez en otros niveles de escolaridad e ingreso, en los ancianos, sus interes y perspectivas.

\section{THE EXPERIENCE OF A GROUP OF RETIRED-ELDERLY PEOPLE: RESEARCH-ACTION}

The present study aims at comprehending the retired people through a group experience as well as allowing them to reflect about their condition. The authors utilized the research-action methodology, with 25 retired subjects who worked in the University, aging from 51 to 79 years old. The narratives related to their experiences were obtained through verbal interaction, group discussions and interviews. From the analysis of the experiences emerged questions associated to the retired-elderly interests, their physical and mental health, relationship, learning process, social environment and leisure.

KEY WORDS: elderly, retired, group of retired-elderly people

\section{REFERÊNCIAS BIBLIOGRÁFICAS}

01. ROGERS, C. Grupos de encontro. 2. ed. São Paulo: Martins Fontes, 1979.
02. THIOLLENT, M. Metodologia da pesquisa: ação.

4. ed. São Paulo: Cortez, 1988. 